\title{
Nintedanib and a bi-specific anti-VEGF/Ang2 nanobody selectively prevent brain metastases of lung adenocarcinoma cells
}

\author{
Bogdana Kovalchuk ${ }^{1,2} \cdot$ Anna S. Berghoff ${ }^{1,2,5} \cdot$ Matthia A. Karreman ${ }^{1,2} \cdot$ Katharina Frey $^{1,2} \cdot$ Manuel Piechutta $^{1,2}$. \\ Manuel Fischer ${ }^{3}$. Julia Grosch ${ }^{1,2}$. Sabine Heiland ${ }^{3} \cdot$ Michael O. Breckwoldt ${ }^{3}$. Frank Hilberg ${ }^{4} \cdot$ Wolfgang Wick $^{1,2}$. \\ Frank Winkler ${ }^{1,2}$ (1)
}

Received: 15 June 2020 / Accepted: 6 September 2020 / Published online: 12 September 2020

(c) The Author(s) 2020

\begin{abstract}
Brain metastases (BM) are an ever-increasing challenge in oncology, threatening quality of life and survival of many cancer patients. The majority of BM originate from lung adenocarcinoma, and stage III patients have a risk of $40-50 \%$ to develop $\mathrm{BM}$ in the first years of disease onset. As therapeutic options are limited, prevention of their occurrence is an attractive concept. Here we investigated whether Nintedanib (BIBF 1120), a tyrosine kinase inhibitor (TKI) targeting the VEGF pathway approved for lung adenocarcinoma, and the dual anti-VEGF-A/Ang2 nanobody BI836880 have the potential to prevent $\mathrm{BM}$ formation. A mouse model of brain metastasis from lung adenocarcinoma was used in which tumor cells were injected intracardially. Metastases formation occurred inside and outside of the brain and was followed by MRI, IVIS, and immunohistochemistry. BM were reduced in volume and number by both Nintedanib and the dual anti-VEGF-A/Ang2 nanobody, which translated into improved survival. Both compounds were able to normalize cerebral blood vessels at the site of brain metastatic lesions. Extracranial metastases, however, were not reduced, and meningeal metastases only partially. Interestingly, unspecific control $\operatorname{IgG}$ also lead to brain vessel normalization and reduction of brain and meningeal metastases. This data indicates a brain-specific group effect of antiangiogenic compounds with respect to metastasis prevention, most likely by preventing an early angiogenic switch. Thus, Nintedanib and BI836880 are promising candidates for future BM preventive study concepts in lung adenocarcinoma patients.
\end{abstract}

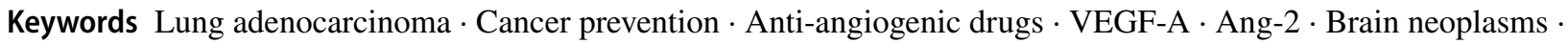
Xenograft metastasis model · Therapeutic IgG

\section{Abbreviations}

Ang2 Angiopoietin 2

ALK Anaplastic lymphoma kinase

Frank Winkler

frank.winkler@med.uni-heidelberg.de

1 Neurology Clinic and National Center for Tumor Diseases, University Hospital Heidelberg, Im Neuenheimer Feld 400, 69120 Heidelberg, Germany

2 Clinical Cooperation Unit Neurooncology, German Cancer Consortium (DKTK), German Cancer Research Center (DKFZ), Heidelberg, Germany

3 Department of Neuroradiology, University Hospital Heidelberg, Heidelberg, Germany

4 Department of Pharmacology, Boehringer Ingelheim RCV $\mathrm{GmbH} \& \mathrm{Co} \mathrm{KG}$, Vienna, Austria

5 Division of Oncology, Department of Medicine I, Medical University of Vienna, Vienna, Austria
BBB Blood brain barrier

BM Brain metastases

BRAF Rapidly accelerated fibrosarcoma isoform B

cMRI Cranial magnetic resonance imaging

DAPI 4',6-diamidin-2-phenylindol

EGFR Epidermal growth factor receptor

GFP Green fluorescent protein

IVIg Intravenous immunoglobulin

IVIS In vivo bioluminescence imaging system by Perkin Elmer

MEK Mitogen-activated protein kinase kinase

ROI Region of interest

RTG Research training group

TKI Tyrosine kinase inhibitor

VEGF Vascular endothelial growth factor 


\section{Introduction}

Brain metastases (BM) have an increasing incidence [1-3] and are associated with high morbidity and mortality, affecting neurological function and quality of life, with a mean overall survival of affected patients of a few months only. Many patients suffering from solid cancers are at high risk of developing BM during the course of their disease. BM occur most frequently in lung adenocarcinoma, where up to $50 \%$ of stage III patients (locally advanced) develop BM within 24 months after "definitive" treatment with surgery, radiation, and chemotherapy $[4,5]$.

The treatment of established, clinically relevant BM is complicated by the fact that the disease is generally multifocal in nature. Moreover, the brain is a difficult, delicate organ where many locally aggressive and systemic therapies are not possible or not effective [3]. One major issue is that most chemotherapeutics fail to pass the blood brain barrier (BBB) and blood tumor barrier in sufficient concentrations [6]. Targeted therapies, however, can be very effective against established BM from different entities, such as BRAF and MEK inhibitors in melanoma, EGFR and ALK inhibitors in lung adenocarcinoma, and immune checkpoint inhibitors in melanoma and lung cancer [3, 7-10]. There is also some promising clinical data for antiangiogenic drugs, particularly bevacizumab, with indications for activity against BM, additional beneficial anti-edema effects, and reduction of radiation-induced necrosis [11-18].

Considering the difficulties of BM treatment, it appears logical that their prevention must be a prime goal of future therapies. Especially patients with a high risk to develop brain metastasis (HER2+ or triple-negative breast cancer, melanoma, small-cell lung cancer and stage III/IV non-small-cell lung cancer (NSCLC) [3]) would benefit from such a preventive intervention. Prophylactic wholebrain radiation therapy has already proven to be effective in reducing BM $[19,20]$, although associated with significant neurotoxicity [19-22]. BM prevention by a non-neurotoxic treatment, ideally a systemic drug that is well tolerated even with long-term administration, is therefore an exciting possibility that however is still in need for a full pre-clinical characterization and clinical validation. Conceptually, the most promising biological approach would be to target BM initiating cancer cells at very early time points of the brain metastatic cascade, e.g. by preventing the mandatory and VEGF-dependent angiogenic switch during micrometastases proliferation in lung adenocarcinoma $[3,15,23]$. The anti-VEGF-A antibody bevacizumab was shown to prevent BM in a lung adenocarcinoma mouse BM model [23], and the retrospective analysis of the AVAIL trial revealed a reduction of BM as first site of relapse with bevacizumab treatment [15]. Moreover, in a prospective clinical trial of stage IV lung adenocarcinoma with mutated EGFR, the addition of bevacizumab to the standard tyrosine kinase inhibitor (TKI) treatment increased progression free survival and reduced $\mathrm{BM}$ formation [24]. It remains unclear whether the brainspecific preventive effect seen in both these studies was an explicit effect of bevacizumab, or rather due to a class effect of antiangiogenic drugs. For a clinical BM prevention concept, oral drugs that can be taken over years in an outpatient setting might be more feasible. In a phase 3 multicenter double-blind randomized trial (LUME-Lung 2 [25]) the oral anti-VEGF pathway TKI Nintedanib has prolonged survival in relapsed or refractory advanced NSCLC.

In this study, the two antiangiogenic compounds Nintedanib and the new dual anti-VEGF/Ang2 nanobody BI836880 were therefore tested for their ability to inhibit BM development and growth, reduce development of extracranial metastases, and prolong survival. For this purpose, a mouse model was selected that reliably reflects the human disease, including frequent development of BM that are relevant for the clinical course after systemic tumor cell inoculation $[15,23]$.

\section{Material and methods}

\section{Murine brain metastasis model}

The brain seeking subline PC14 -PE6 pGF1 Br4 of the human lung adenocarcinoma cell line PC14-PE6 was used to model brain metastasis formation in immunodeficient NOD/SCID mice, as described by Ilhan-Mutlu et al. [15]. To receive an even higher brain affinity and number of BM, two additional reinjections of successfully brain-metastasized cells, as described before [15], were performed and the PC14-PE6 pGF1 Br4 cell line was obtained from the previously used PC14-PE6 pGF1 Br2 cell line. This so called "brain training" does increase the chance of metastatic cells to form metastases in the brain and does not primarily inhibit metastasis formation to other organs [26].

Briefly, PC14-PE6 pGF1 Br4 cells were trypsinized (Gibco, Life Science Technologies, cat. no.: 25200-056), washed, counted and resuspended in PBS (cat. no: D8537, Sigma Life Sciences) in a final concentration of $5 \times 10^{6}$ cells/ mL. 8-week-old male NOD/SCID mice were anesthetized with a xylazine-ketamine-injection (intraperitoneal injection of $100 \mu \mathrm{L} \mathrm{NaCl}$-solution containing $1 \mathrm{mg} / \mathrm{mL}$ of xylazine (Rompun $2 \%, 20 \mathrm{mg} / \mathrm{mL}$, Bayer) and $15 \mathrm{mg} / \mathrm{mL}$ ketamine $(100 \mathrm{mg} / \mathrm{mL}, \mathrm{WDT}))$. Thereafter $5 \times 10^{6}$ PC14-PE6 pGF1 $\mathrm{Br} 4$ cells $(100 \mu \mathrm{L})$ were singularized through a filter tube (BD-Falcon, BD Biosciences, cat. no: 352235) and injected 
in into the left cardiac ventricle with a $30 \mathrm{G}$ needle. Through blood-circulation tumor cells are then distributed into the organs. All animals were handled according to the German animal protection law (Approving institution: Regierungspräsidium Karlsruhe).

Cells were cultured in DMEM (PAN Biotech, cat. no: P04-03600, $500 \mathrm{~mL}$ ) containing $4.5 \mathrm{~g} / \mathrm{L}$ glucose, sodium pyruvate, $3.7 \mathrm{~g} / \mathrm{L} \mathrm{NaHCO} 3$ without L-glutamine supplemented with $10 \%$ heat-inactivated FBS (Sigma-Aldrich, cat. no: F7524), $1 \mathrm{~mL}$ penicillin/streptomycin (SigmaAldrich, cat. no: P4333), and $5 \mathrm{~mL}$ of Glutamax (Gibco, Life Science Technologies, cat. no.: 25200-056) in a humidified atmosphere of $10 \% \mathrm{CO} 2$ at $37{ }^{\circ} \mathrm{C}$. They were passaged via trypsinization (Gibco, Life Sciences, cat. no: $25200-056$ ) when reaching $90 \%$ of confluence. Transduced with a pGF1-CMV reporter, the used cells express both, copGFP and firefly luciferase. Thus, FACS sorting of GFP-expressing cells was performed (on FACSAria1, BD Biosciences) prior to cell expansion for injection. Furthermore, cell line authenticity was confirmed using a Multiplex human cell line authentication test, which is provided by Multiplexion.

\section{Treatment protocol}

To evaluate different antiangiogenic compounds, mice were randomized to four separate intervention groups with 12 mice per group (control IgG group $n=14$ ). Treatment started one day prior to heart injection to ensure full BM preventive activity and was always adapted to body weight.

The first group received daily treatment with Nintedanib (BIBF 1120, Boehringer-Ingelheim) in comparison to its control group, receiving $200 \mu \mathrm{L}$ of carrier solution (0,5\%-Hydroxyethylcellulose, cat. no.: 822068, Merck) only. Nintedanib is a triple angiokinase inhibitor blocking VEGFR, PDGFR and FGFR kinase activity and was shown to reduce vessel density and vessel integrity in human tumor xenografts [27]. It was solved in $0,5 \%$-Hydroxyethylcellulose (final concentration $5 \mathrm{mg}$ / $\mathrm{mL}$ ) and applied via oral gavage in a dosage of $50 \mathrm{mg} / \mathrm{kg}$ (ca. $200 \mu \mathrm{L}$ per mouse).

The third group was treated every 3rd day with a combined anti-VEGF and anti-Ang2 nanobody (BI836880, MW appr. $40.7 \mathrm{kDa}$; obtained by Boehringer-Ingelheim) in contrast to its respective control group (fourth group), which received a control antibody (InVivoMAb rat IgG2a isotype control, MW $150 \mathrm{kDa}$; BioXCell) of equal dosage, frequency and concentration. Nanobody and control antibody were solved in sterile PBS (cat. no: D8537, Sigma Life Sciences) reaching a concentration of $2.615 \mathrm{mg} / \mathrm{mL}$, their application dose was $15 \mathrm{mg} / \mathrm{kg}(100-150 \mu \mathrm{L}$ per mouse).

\section{In vivo bioluminescence imaging (IVIS)}

Metastasis development was monitored by in vivo bioluminescence imaging (IVIS Lumina Series III Imaging system, PerkinElmer) on day 1 (baseline imaging), day 14 and day 28 after tumor cell injection. For image acquisition the mice received an intraperitoneal injection of Luciferin (Luciferin substrate cat. no.: 5306500001, Calbiochem; dosage: $150 \mathrm{mg} / \mathrm{kg}$; stock solution: $30 \mathrm{mg} / \mathrm{mL}$ in $\mathrm{H}_{2} \mathrm{O}$; application volume: $100-150 \mu \mathrm{L}$ ). After 3 min of incubation the animals were sedated with 5\% isoflurane and then transferred to the imaging chamber with $2 \%$ isoflurane and $37^{\circ} \mathrm{C}$. Imaging was started 10 min after Luciferin injection using the XFOV24 lense and an exposure time of $180 \mathrm{sec}$ (medium bining, $1.2 \mathrm{~F} / \mathrm{Stop}$, minimum target count luminescent: 10,000). Images were taken from the ventral as well as from the dorsal view.

\section{In vivo cranial MRI}

For more precise in vivo evaluation of intracranial metastases formation, cranial MRI (cMRI, 9.4 T, Bruker Topspin 9/20) after Gadolinium contrast administration was performed on day 26 after intracardial tumor cell injection. Mice were sedated with $3 \%$ isoflurane and kept under anesthesia at $0.5-1.5 \%$. Constant body temperature was maintained at $37{ }^{\circ} \mathrm{C}$ by a heating plate. During imaging respiration was surveilled using an external breathing surface pad (in house development, LabVIEW program, National Instruments Corporation). A dose of $0.2 \mathrm{mmol} / \mathrm{kg}$ i.v. gadodiamide (Omniscan; Nycomed) was given to each animal and standard T1-w and T2-w images were acquired. For quantification of tumor volumes, tumors were manually segmented on T1-w images using the Fiji software (general public license) [28].

\section{Follow up and organ preservation}

To prevent confounding and observer bias, mice of different intervention groups were hold together in common cages and were distinguished by small ear punches, visible only at a very close look. Daily control for adverse events, including neurological symptoms, poor outer appearance and weight loss of more than $20 \%$, was performed. At the occurrence of one of the latter the animals were sacrificed to prevent suffering and their lifetime was documented for survival analysis. A left cardiac perfusion with PBS (cat. no: D8537, Sigma Life Sciences) and, subsequently, 4.5\% paraformaldehyde (Roti-Histofix, cat. no: 22135, ROTH) was performed. After $1 \mathrm{~h}$ of paraformaldehyde fixation at room temperature, the brain tissue was incubated in $30 \%$ sucrose solution (cat. no: 84097-1 KG, Sigma Life Sciences, diluted in PBS) at $4{ }^{\circ} \mathrm{C}$ for $24 \mathrm{~h}$ and preserved at $-80^{\circ} \mathrm{C}$, embedded 
into optimal cutting temperature medium (Roti-Histofix, cat. no: $22135, \mathrm{ROTH})$.

\section{Histology and immunostaining}

To prepare slides for histology, brain tissue of 6 mice per group (randomly chosen to prevent observer bias) was cut into $12 \mu \mathrm{m}$ thick coronary cryo-sections with a layer distance of $1000 \mu \mathrm{m}$ (cryostat Leica CM3050 S). Vascular basement membrane staining was performed as described previously $[15,29]$ using an anti-collagen-IV primary antibody $(1: 200$, rabbit anti-collagen type IV, cat. no.: AB756P, Merck) and a fluorescent secondary antibody $(1: 400$, goat anti-rabbit AlexaFlour 546, cat. no.: A11010, life). Briefly, slides were dried under air flow (10 min), washed with ice-cold acetone $(1 \times 10 \mathrm{~min})$ following PBS $(3 \times 5 \mathrm{~min})$ and then delineated with an invisible fat marker (Dako Pen, cat. no: 52002). After incubation with blocking buffer $(1 \times 30 \mathrm{~min}$; blocking buffer $=10 \%$ donkey serum in TBST buffer (Tris-buffered saline, $0.1 \%$ Tween 20 cat.no.: 52194-1 g, Sigma) the primary antibody was applied, and the samples were incubated at $4{ }^{\circ} \mathrm{C}$ overnight. The next day they were washed with PBS $(3 \times 5 \mathrm{~min})$ and covered with secondary antibody for $1 \mathrm{~h}$. Finally, the slides were washed with PBS $(3 \times 5 \mathrm{~min})$ and shielded with a drop of Vectashield mounting medium with DAPI (Vector Laboratories, cat. no: H-1500) and a cover slip before their storage at $4{ }^{\circ} \mathrm{C}$.

Images for the analysis of metastatic foci were acquired by a brightfield slide scanner (Zeiss Axio Scan. Z1, magnification: $\times 20$, tile-scans of the whole section area), whereas images for vascular basement membrane assessment were taken confocally (Zeiss LSM 710 ConfoCor 3, magnification $\times 40$ ). Thereby, high resolution images of three different maximally vascularized areas were recorded, both inside and outside of metastatic foci. All images were taken with identical acquisition settings and GFP, DAPI and Alexa Flour 546 signal was detected.

\section{Image analysis}

In histology analysis, metastatic events were counted manually, using the ZEN lite software (Zeiss, blue edition). GFP positive metastatic foci were easily detectable by fluorescence microscopy [23, 30]: To quantify the thickness of collagen-IV positive vascular basement membrane $4 \times 4$ grids were superimposed onto $300 \mu \mathrm{m} \times 300 \mu \mathrm{m}$ large confocal images as described previously [29]. Diametral thickness was measured wherever Alexa Flour 546 positive structures and grid lines intersected. If less than 6 intersections occurred, $6 \times 6$ grids were used. Area measurements were performed with the Fiji software measurement tool, after converting the images to RGB and selecting the area of GFP positive foci with the color threshold tool. Non-parenchymal meningeal metastases were excluded from area measurements by the selection tool. As scaling information was changed when transferring the images from ZEN to Fiji, pixel size properties in Fiji were adapted to fit the actual scale parameters recorded in ZEN.

Original cMRI data were converted into Fiji stacks and metastasis volume was measured using the segmentation editor and 3D manager plugins. Metastases were depicted manually on T1-w images by their hyperintense spherical character. For a separate investigation of meningeal and parenchymal metastases, lesions with any connection to the meningeal space were defined as meningeal metastases in contrast to parenchymal metastases, which needed to be surrounded by brain parenchyma only.

For IVIS evaluation, Living Image Software Version 4.4 (PerkinElmer) was used and time-sequences of each mouse were created. Consequently, total photon flux $(\mathrm{p} / \mathrm{s})$ was quantified, using the baseline image of day 1 to define background ROIs. For each mouse, a region of interest (ROI) was defined, covering the extracranial part of the body from the ears to the tail root. Ventral and dorsal view were quantified separately.

\section{Statistical analysis}

Statistical analysis was performed using GraphPad Prism Software. Survival time was analyzed with Kaplan-Meier survival curves and Gehan-Breslow-Wilcoxon tests. Development of brain metastases was compared by Fisher's exact test. Differences in number and volume of metastases were tested with the Mann-Whitney-U test, and this test was also used to determine extracranial photon flux in IVIS and the thickness differences of the vascular basement membrane in immunohistochemistry.

\section{Results}

\section{Nintedanib and an anti-VEGFA/Ang2 nanobody prevent brain metastases and improve survival}

The potential of both Nintedanib, a clinically approved TKI for lung adenocarcinoma, and an investigational dual antiVEGF/Ang2 nanobody (BI 836880) to prevent the occurrence of clinically relevant $\mathrm{BM}$ was investigated. A brainseeking PC14-PE6 human lung adenocarcinoma subline (PC14-PE6 pGF1 Br4) was selected, which robustly generates a sufficient number of BM for analysis, but to a lesser extent also extracranial metastasis in lung, bone and other organs. Importantly, the results obtained with this cell line demonstrated a high level of concordance between mouse studies and analysis of patient data with respect to patterns of brain and systemic metastasis development [15, 23]. Mice 


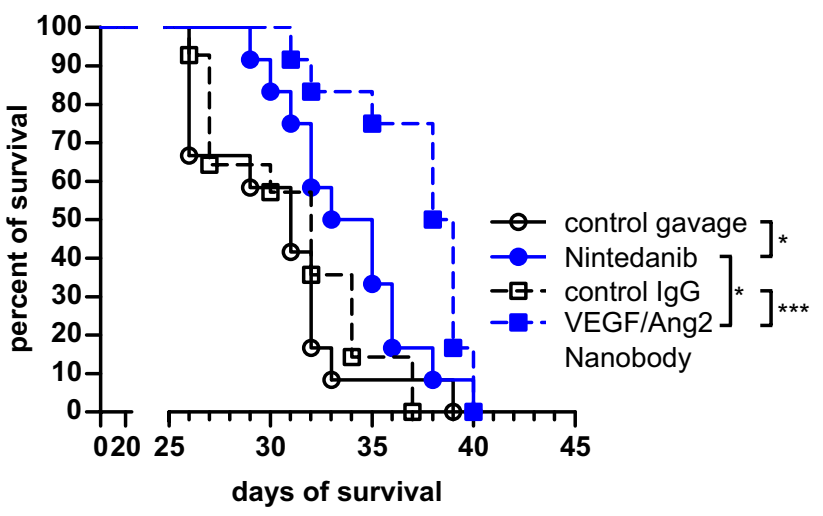

Fig. 1 Nintedanib and VEGF/Ang2 nanobody prolong animal survival. Kaplan-Meier survival curves of the four treatment groups receiving control gavage, Nintedanib, control IgG, or VEGF/Ang2 nanobody. Median survival: 32 days; $\mathrm{n}=12$ mice per group, except of control IgG group $\mathrm{n}=14$. $* \mathrm{p}<0.05$ and $* * * \mathrm{p}<0.001$ Gehan-Breslow-Wilcoxon test

were divided into four treatment groups, receiving either Nintedanib vs. control gavage, or anti-VEGF/Ang2 nanobody vs. control antibody treatment during the metastatic process.

Both Nintedanib and the anti-VEGF/Ang2 nanobody prolonged the survival of metastases-bearing animals (Fig. 1; $p=0.0246$ for Nintedanib, $p=0.0003$ for anti-VEGF/Ang2 nanobody, Gehan-Breslow-Wilcoxon test), with the antiVEGF/Ang2 nanobody being even more effective than Nintedanib ( $p=0.017$, Gehan-Breslow-Wilcoxon test). First neurological symptoms occurred on day 26 after tumor cell injection, mostly followed by a rapid disease progression. The maximum survival was 40 days, when the last animals were sacrificed according to animal regulations. Evaluation with high field $9.4 \mathrm{~T}$ cranial MRI (cMRI) on day 26 revealed that $75.0 \%(9 / 12)$ of mice with Nintedanib treatment developed detectable brain parenchymal metastases $(\mathrm{BM})$, in contrast to $100 \%(12 / 12)$ in their matched control (Fig. 2a). Strikingly, only 16.7\% (2/12) of VEGF/Ang2 nanobody treated mice developed radiologically detectable $\mathrm{BM}$, in comparison to $64.3 \%$ (9/14) in the control antibody group ( $\mathrm{p}=0.0214$, Fisher's exact test), supporting the BMpreventive potential of nanobody-treatment. Interestingly, when directly comparing the control groups, the control $\operatorname{IgG}$ group had significantly less BM than the group receiving control carrier solution via oral gavage $(\mathrm{p}=0.0425)$.

\section{Nintedanib and nanobody reduce metastasis formation specifically in the brain}

We further aimed to investigate if prolonged overall survival was not only a result of the reduced number of mice developing clinically relevant brain metastases, but also a decrease in number and size of the BM. Using Gadolinium contrast cMRI at day 26 post intracardial tumor cell injection, metastatic lesions were detected and quantified (Fig. 2b). Both Nintedanib and the anti-VEGF/Ang2 nanobody significantly reduced the number $(\mathrm{p}=0.0123$ for Nintedanib and $\mathrm{p}=0.0059$ for nanobody, Mann-Whitney-U test) and volume ( $p=0.0006$ for Nintedanib and $p=0.0055$ for nanobody, Mann-Whitney-U test) of brain parenchymal metastases (Fig. 2c). Furthermore, Nintedanib also decreased the volume but not the number of meningeal metastases (Fig. 2d; p =0.0459, Mann-Whitney-U test), while the anti VEGF/Ang2 nanobody did not $(\mathrm{p}=0.9758$, Mann-Whitney-U test). Again, number and volume of meningeal metastases were significantly reduced, when comparing the unspecific IgG control with the oral gavage control group (Fig. 2d, p $=0.0057$ (number) and $\mathrm{p}=0.0007$ (volume), Mann-Whitney-U test).

Next, we sought to investigate whether the antimetastatic activity of Nintedanib and nanobody also applies to extracranial sites by using whole-body imaging (IVIS). Neither Nintedanib nor nanobody were able to reduce the burden of extracranial metastatic disease (Fig. 3, $p=0.2318$ for Nintedanib, $p=0.8490$ for nanobody; Mann-Whitney-U test). Furthermore, the number of extracranial metastases did not differ between treatment groups $(p=0.3797$ for Nintedanib and $p=0.2084$ for nanobody; Mann-Whitney-U test, data not shown). Together these data suggest that both Nintedanib and nanobody prevent metastases outgrowth in the brain, but not relevantly outside the CNS.

\section{Nintedanib and nanobody, but also IgG normalize blood vessels in brain metastases}

Antiangiogenic therapies, particularly VEGF pathway inhibition, are known to reduce the abnormally thickened vascular membrane to more normal levels, which is strongly associated with improved vascular morphology and function, and finally response to irradiation in angiogenic brain tumor models [29]. To determine the vascular basement membrane thickness, we used anti-collagen IV staining as described before [29]. As expected, within metastatic foci of control mice, the vascular BM showed abnormal thickness and organization, while a very thin, linear basement membrane was found in the normal brain parenchyma (Fig. 4a). In contrast, the vascular basement membrane after Nintedanib and anti-VEGF/Ang2 nanobody treatment appeared almost normal inside of metastatic foci. (Fig. 4b, p $<0.0001$ for both, Mann-Whitney-U test). Interestingly, also unspecific control IgG treatment lead to vessel normalization $(\mathrm{p}<0.0001$, Mann-Whitney-U test). In normal brain tissue, no difference between the four treatment groups was detectable (Fig. 4c). 
a

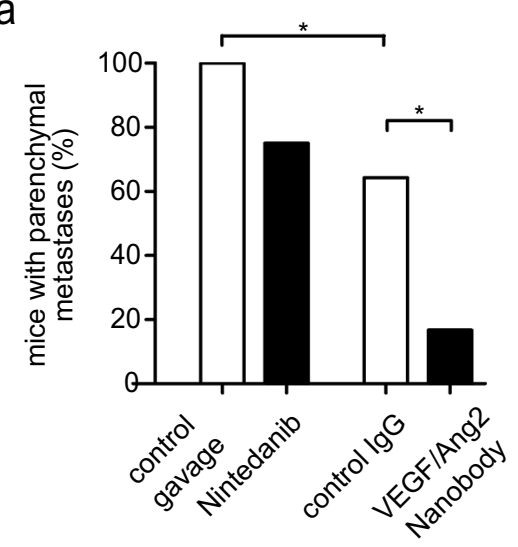

C
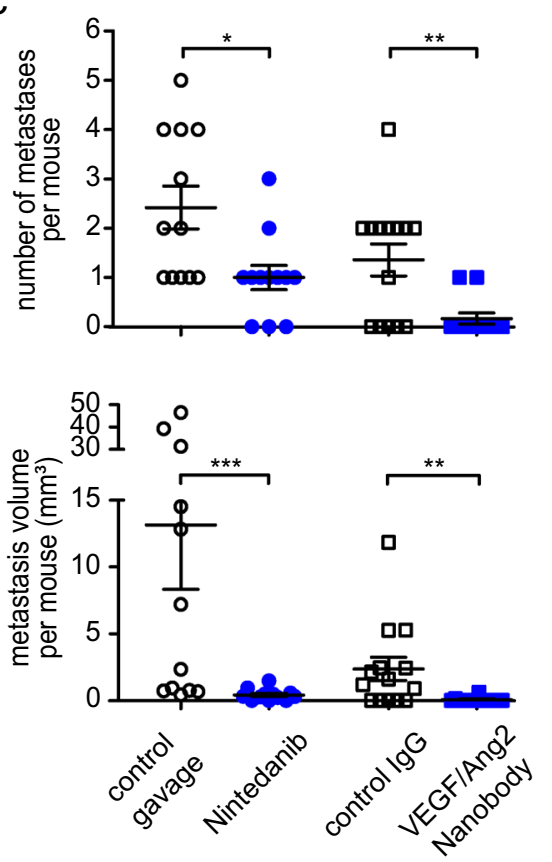

f

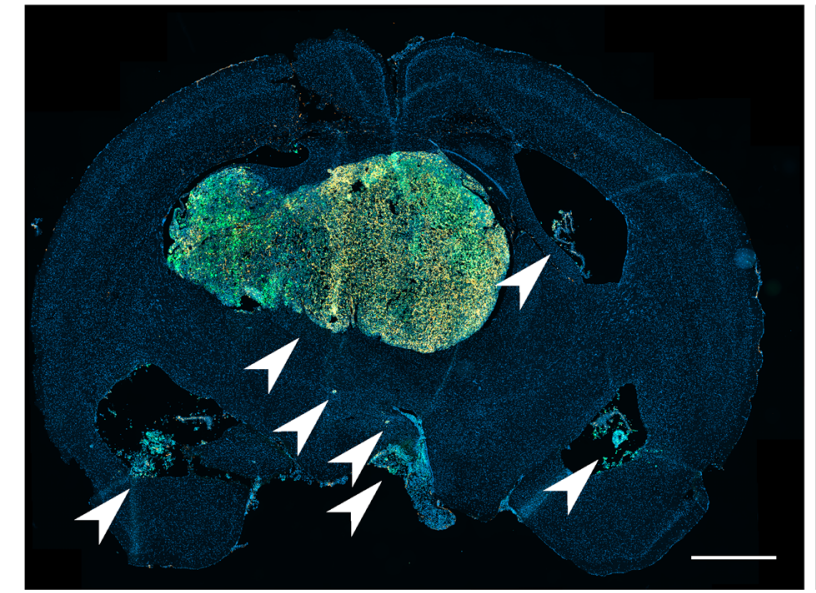

control gavage b
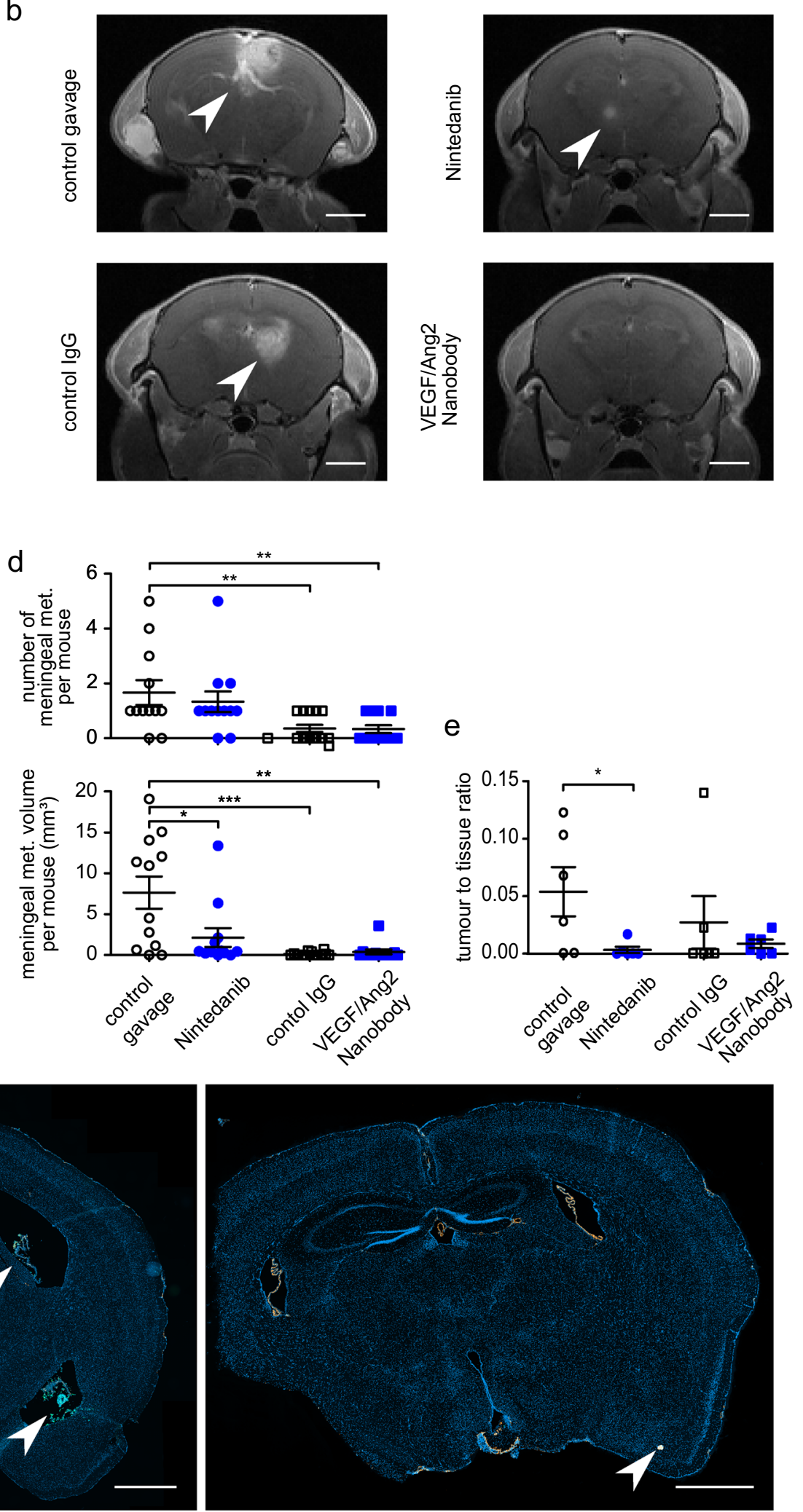

Nintedanib 
४Fig. 2 Nintedanib and anti-VEGF/Ang2 nanobody prevent brain metastases formation. 9.4 T MRI after Gadolinium contrast administration was performed on day 26 after intracardial tumor cell injection. a Bar chart illustrating the reduced percentage of mice with metastases, detectable in cMRI. b Representative T1-w cMRI images depicting larger intracranial metastases in the control groups, indicated by arrowheads. scale bar $=2 \mathrm{~mm}$. c Scatter plots showing the reduction of number and volume of cranial metastases in cMRI. d Scatter plots depicting the number and volume of meningeal metastases in cMRI e Quantification demonstrating a higher histological tumor-tissue ratio in control mice at their time of death. $\mathbf{f}$ Representative histological slices with higher number and size of PC14-PE6 pGF1 Br4 metastases in the control group. Fluorescent staining: DAPI $($ blue $)=$ nucleus, GFP $($ green $)=$ PC14-PE6 tumor cells, Alexa Flour 546 (orange) $=$ collagen-IV positive vascular basement membrane. Arrows indicate GFP-positive metastatic lesions. Scale bar $=1000 \mu \mathrm{m}$. Mean values with standard errors of the mean are shown. $* \mathrm{p}<0.05, * * \mathrm{p}<0.01, * * * \mathrm{p}<0.001$, Mann-Whitney-U test. (Color figure online) a

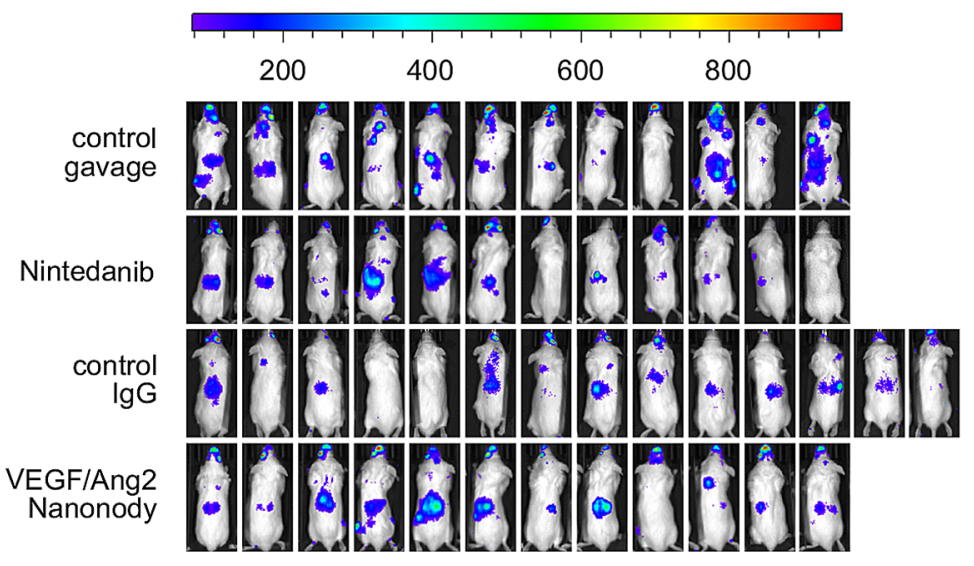

b

Luminescence (counts)
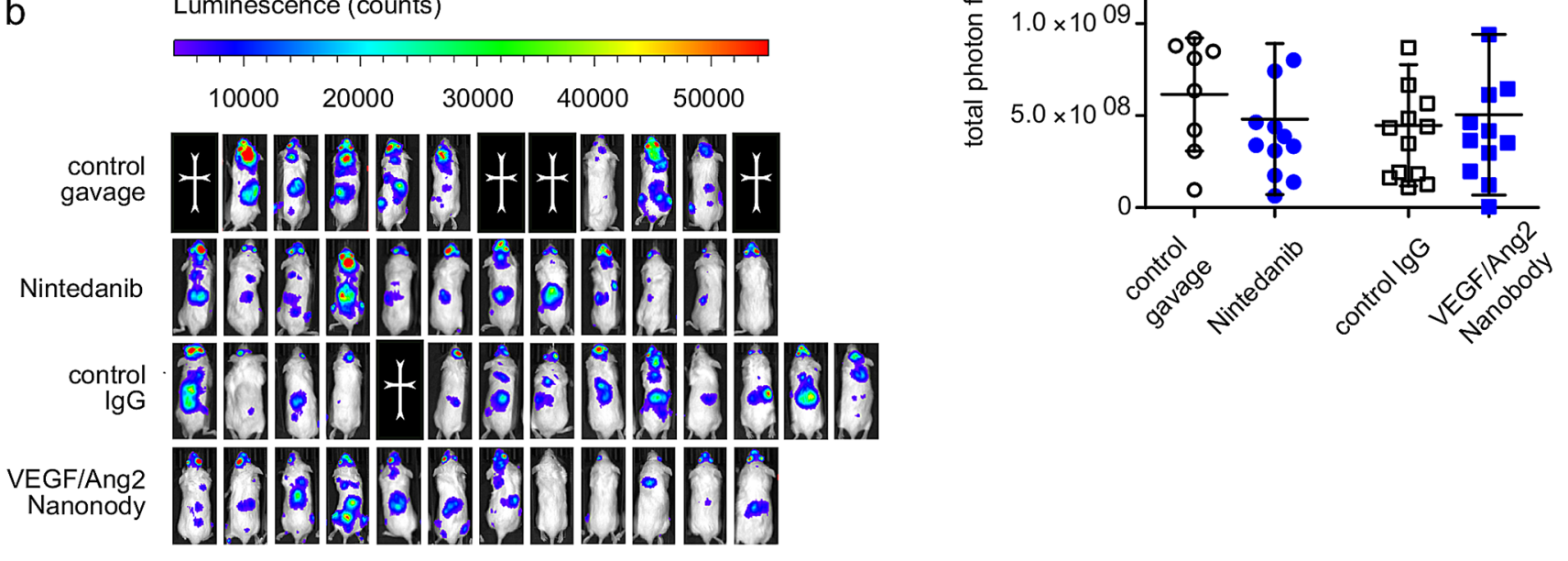

\section{Discussion}

In this study we examined if two antiangiogenic compounds, Nintedanib and the novel dual anti-VEGF/Ang2 nanobody BI836880, were able to prevent brain and extracranial metastases and improve survival outcomes. We found that Nintedanib and the anti-VEGF/Ang2 nanobody prolonged animal survival and reduced BM formation, while extracranial metastases were not reduced. A normalization of vascular basement membrane inside of metastatic lesions was seen in both treatment groups, but also with control IgG, which also prevented BM formation.

In accordance with the effects described for bevacizumab [15], Nintedanib and the anti-VEGF/Ang2 nanobody showed a brain-specific metastasis-preventive effect. This provides further support for the notion that antiangiogenic compounds in general might be predominantly effective in the brain, as $\mathrm{BM}$, especially in lung adenocarcinoma, show a particularly stronger angiogenic reaction at this site [23, 31-33].
Fig. 3 Extracranial systemic metastases are not reduced by antiangiogenic treatments. a, b IVIS luminescence images on day 14 (a) and day 28 (b) after tumor cell heart injection. Photon count visualized

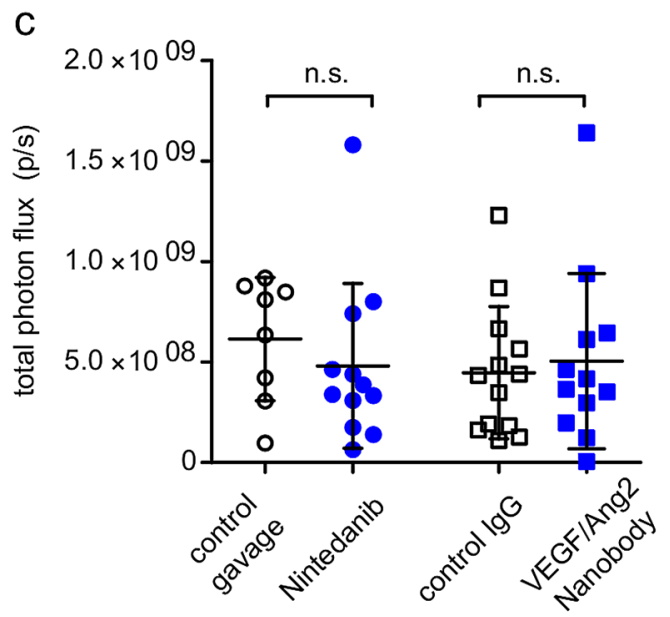

by heat map. c Scatter plot quantifying the extracranial photon flux on day 28 shows no difference between the treatment groups 
a

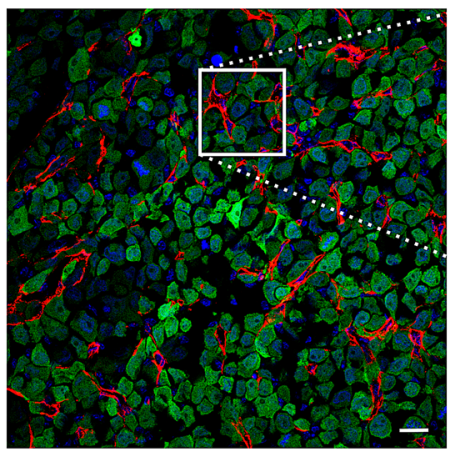

control IgG
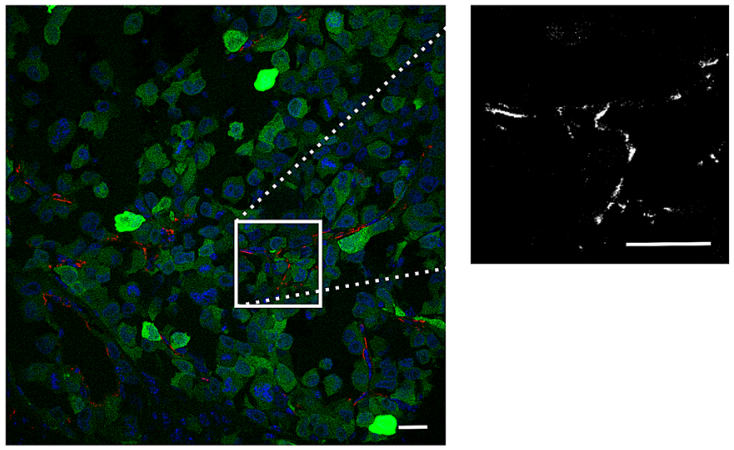

healthy brain tissue
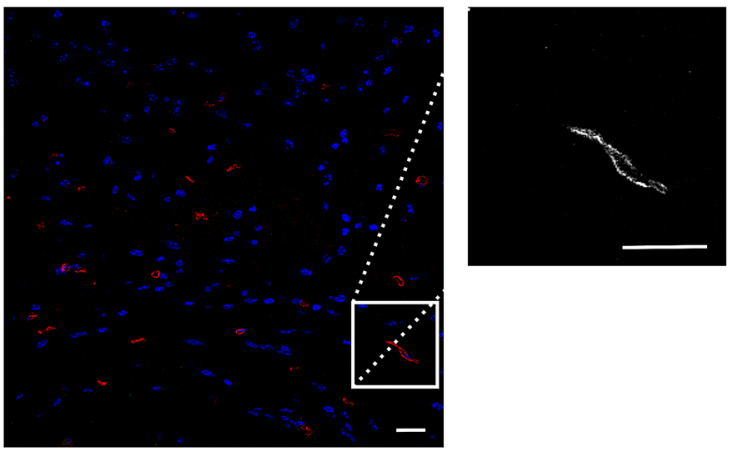

b

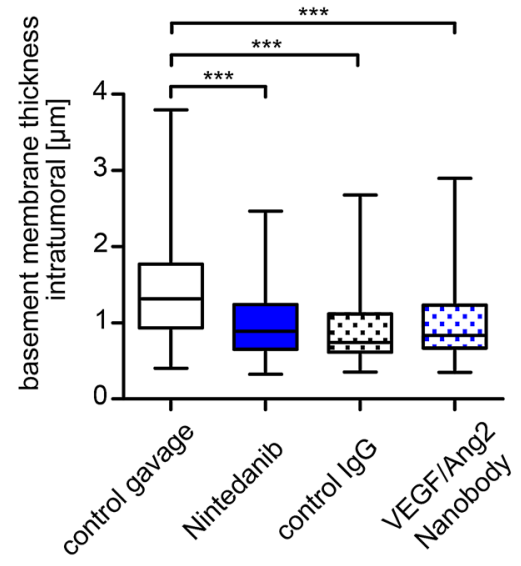

Nintedanib

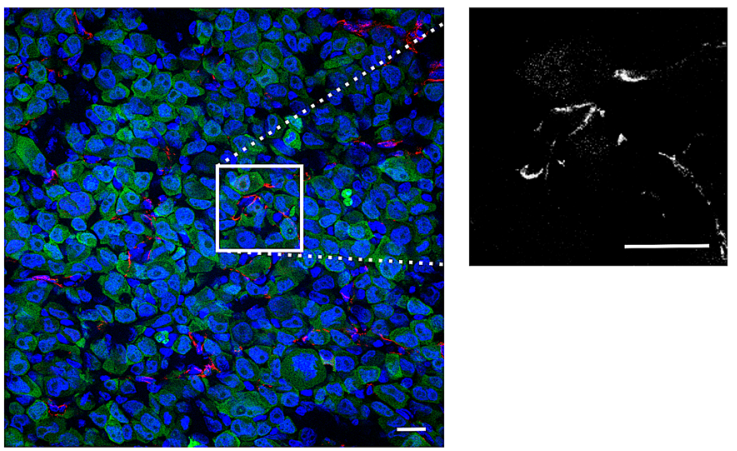

VEGF/Ang2 Nanobody
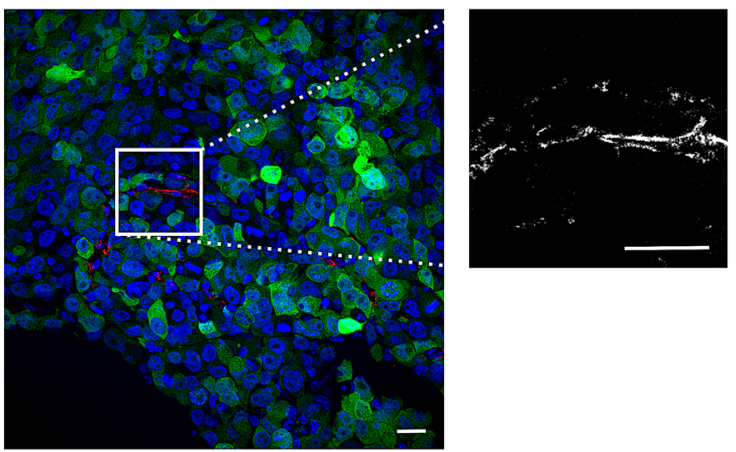

C

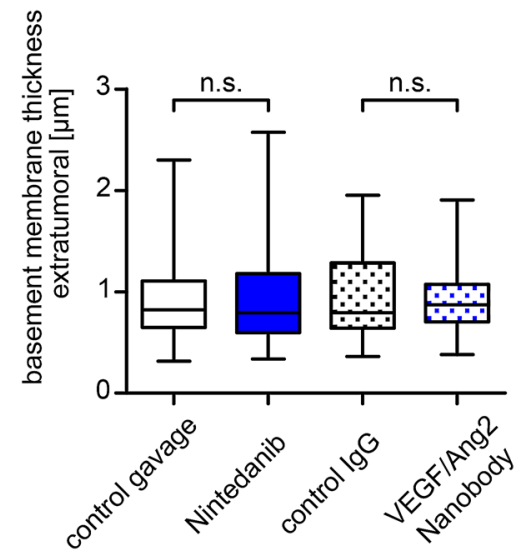


४Fig. 4 Effects of drug treatment on tumor and brain microvessels. a Collagen IV basement membrane staining. Confocal images of representative intratumoral regions of mice from the four treatment groups at time of death. One extratumoral image of healthy brain tissue is also displayed. Nuclear DAPI staining is shown in blue, collagen IV staining in red (Alexa Flour 546) and GFP-expressing PC14-PE6 tumor cells in green. Vascular basement membrane signal (collagen IV) is additionally shown in single-channel images. Scale bar $=20 \mu \mathrm{m}$, magnification 40x. Brightness adjustments were applied equally to all images. b Scatter plot of collagen IV immunostaining inside of metastatic brain lesions. c Scatter plot of collagen IV staining of healthy brain tissue. 3 regions per mouse in 3 mice per group were analyzed. $* * * \mathrm{p}<0.001$, Mann-Whitney-U test. Whiskers indicate minimum and maximum values. (Color figure online)

A recent clinical study confirms that bevacizumab, even as monotherapy (which is not relevantly active outside the brain), has a meaningful clinical activity in advanced brain metastasis, including in lung adenocarcinoma patients [34].

This specific prevention of BM was the most likely cause for the prolonged survival in the treatment groups: survival is governed by the development of BM in the used model [15]. Both Nintedanib and the anti-VEGF/Ang2 nanobody seem to have a comparable overall effectiveness when it comes to BM prevention, however showing a slightly different prevention pattern. While Nintedanib was more efficient in reducing BM volume, the dual antiVEGF/Ang2 nanobody had a stronger preventive effect on metastasis number, which also resulted in a better survival outcome. Considering the relevance of both the early VEGF-dependent angiogenic switch and later angiogenesis-dependent growth for BM formation in lung adenocarcinoma $[3,15,23]$, these findings can best be explained by an earlier activity of the anti-VEGF/Ang2 nanobody compared to Nintedanib when it comes to interference with the brain metastatic cascade.

The use of a brain seeking lung adenocarcinoma subline might have emphasized the significant survival benefit observed here. In fact, this even makes the model more realistic and relevant for the clinical situation, as under current chemotherapeutics and targeted therapies, patients with advanced lung adenocarcinoma still show an elevated incidence of BM: $42 \%$ (Taxan-based therapy) [4] to more than 50\% (Crizotinib) [5] of patients develop BM within 24 months. Furthermore, the site of distant relapse in this patient population is most frequently the brain, and one third of this patient population dies because of a brain specific progress [35]. Together these facts make the development of new BM preventive treatments even more important for lung adenocarcinoma.

Unexpectedly, control IgG treatment showed similar basement membrane normalization effects as Nintedanib did, compared to control gavage treatment. Recently, syngeneic low dose $\mathrm{IgG}$ was shown to counteract cancer progression in melanoma, colon cancer and breast cancer mouse models, inhibiting tumor vessel proliferation and prolonging survival [36]. Anti-tumor effects of unspecific IgG have also been reported using human IgG in animal models [36, 37]. In several case reports, cancer patients who received intravenous immunoglobulin (IVIg) therapy e.g. because of a simultaneous autoimmune disease showed a significant tumor regression, too [38-40]. In our study, unspecific IgG was able to decrease the incidence of meningeal metastases, and a trend to a lower incidence of BM was observed. However, this did not translate into a survival benefit. Taken together this suggests a non-epitope specific antiangiogenic and thus metastasis-preventive mode of action of $\mathrm{IgG}$, at least with respect to the formation of intracranial metastases. This could indeed be relevant for antibody therapies in general, as the ratio of given and endogenous $\mathrm{IgG}$ in the current study (murine $\mathrm{IVIg} / \mathrm{endoIgG}$ ratio $=3.43-1.37$ ) is comparable to the ratios of therapeutically applied and endogenous IgG during e.g. intravenous immunoglobulin therapy for several diseases in humans (IVIg/endoIgG ratio $=2.67-0.9$ ) [41]. An antiVEGF specific activity of IVIg has also been proposed by Damianovich et al. [42]. In a mouse model, an inhibition of melanoma and sarcoma lung metastases was shown by IVIg treatment as well as a prolongation of survival time [43]. Furthermore, it was suggested that IVIg-treatment impairs metastasis and tumor growth by promoting the M1 polarization state of tumor associated myeloid cells, which was associated with a decrease of lung metastatic foci in a melanoma mouse model [44]. Finally, it is an interesting question whether a general BM-preventive effect of the IgG molecule was the reason for the survival benefit of the intravenous anti-VEGF/Ang2 nanobody vs. the orally administered small molecule Nintedanib.

To further clarify the clinical potential of BM prevention by antiangiogenics, randomized controlled clinical trials (minimum stage II) are needed, investigating BM incidence as primary and survival and quality of life as secondary endpoints [3]. Stage III lung adenocarcinoma patients without detectable BM and no active extracranial tumor disease might be the most interesting patient population for such a study [3].

Some limitations exist. It needs to be acknowledged that the rat IgG2a used here is not the absolute appropriate control for a nanobody. Its molecular weight is about 3 times higher, since the nanobody in contrast consists of two variable antibody domains and an albumin module for half-life extension only and lacks the Fc antibody fragment [45]. However, so far there is no nanobody that would not interact with another target protein, so this was the only control compound that came into question. Moreover, a wellcharacterized lung adenocarcinoma cell line which is known to grow particularly angiogenesis-dependent in the brain was used in this study. The effect of an anti-VEGF/Ang2 nanobody in brain metastases on other lung adenocarcinoma cell 
lines needs to be evaluated. However, the simultaneous use of bevacizumab with an Ang2 inhibitor has been shown to reduce brain metastasis in the breast cancer cell lines MDAMB-231br and 4T1br [46], so it is likely that the results of the current study can be more widely generalized to other cell lines and clinical situations. Finally, the immunodeficient mouse models that are the ones that are available today for research on the formation of blood-borne brain macrometastases [26] do not allow for conclusions about the immune microenvironment of metastatic lesions, which is another limitation of this study.

In summary, this study demonstrates that the TKI Nintedanib, an anti-VEGFA/Ang2 nanobody, but to a lesser extend also unspecific $\mathrm{IgG}$ can reduce BM formation, making blood vessel stabilization an attractive mechanism of $\mathrm{BM}$ prevention in lung adenocarcinoma. While the prime time of antiangiogenesis in oncology is fading, BM and their prevention emerge as promising targets that have not been adequately explored yet. The high effectivity against BM formation reported here also fits well to the general concept that the activity of a given drug, or class of drugs, can be very different when comparing effects in the setting of metastases prevention vs. effects on the primary tumor [13, $14,47-50]$.

Acknowledgements Nintedanib (BIBF 1120) and BI 836880 were kindly provided by Frank Hilberg (Boehringer Ingelheim), confocal and widefield imaging devices and technical support by Damir Krunic (DKFZ imaging core facility), and IVIS by the animal core facility of DKFZ.

Author contributions Conception and design: ASB, FH, MAK, WW and FW; Development of methodology: BK, AI-M and FW; Acquisition, analysis and interpretation of data (e.g., statistical analysis, biostatistics, computational analysis): BK, KF, MP, MF, JG, MOB and FW; Writing, review, and/or revision of the manuscript: BK, ASB, MAK, KF, MP, MF, JG, SH, MOB, FH, WW and FW; Administrative, technical or material support: JG, AI-M, ASB, MAK, MB, FH, WW, FW and SH.

Funding Open Access funding enabled and organized by Projekt DEAL. This study was funded by the Deutsche Krebshilfe (German Cancer Aid), Priority Program "Translational Oncology", \#70112507, "Preventive strategies against brain metastases" and the Deutsche Forschungsgemeinschaft (DFG, German Research Foundation)Project number 259332240/RTG 2099. M.O.B. was supported by a physician-scientist fellowship of the Medical Faculty, University of Heidelberg and the Else Kröner-Fresenius Stiftung (2017-A25).

\section{Compliance with ethical standards}

Conflict of interest FW received a research grant from Boehringer for conducting this work. FW also received research grants from Roche, Genentech, GSK, and Divide \& Conquer Ltd. ASB, MOB, MAK, MP and BK have no conflict of interest to disclose.

Ethical approval All applicable international, national, and/or institutional guidelines for the care and use of animals were followed. All procedures performed in studies involving animals were in accordance with the ethical standards of the institution or practice at which the studies were conducted.

Open Access This article is licensed under a Creative Commons Attribution 4.0 International License, which permits use, sharing, adaptation, distribution and reproduction in any medium or format, as long as you give appropriate credit to the original author(s) and the source, provide a link to the Creative Commons licence, and indicate if changes were made. The images or other third party material in this article are included in the article's Creative Commons licence, unless indicated otherwise in a credit line to the material. If material is not included in the article's Creative Commons licence and your intended use is not permitted by statutory regulation or exceeds the permitted use, you will need to obtain permission directly from the copyright holder. To view a copy of this licence, visit http://creativecommons.org/licenses/by/4.0/.

\section{References}

1. Nayak L, Lee EQ, Wen PY (2012) Epidemiology of brain metastases. Curr Oncol Rep 14(1):48-54. https://doi.org/10.1007/s1191 2-011-0203-y

2. Bachmann C, Schmidt S, Staebler A, Fehm T, Fend F, Schittenhelm J, Wallwiener D, Grischke E (2014) CNS metastases in breast cancer patients: prognostic implications of tumor subtype. Med Oncol 32(1):400. https://doi.org/10.1007/s12032-014-0400-2

3. Valiente M, Ahluwalia MS, Boire A, Brastianos PK, Goldberg SB, Lee EQ, Le Rhun E, Preusser M, Winkler F, Soffietti R (2018) The evolving landscape of brain metastasis. Trends Cancer 4(3): 176196. https://doi.org/10.1016/j.trecan.2018.01.003

4. Mamon HJ, Yeap BY, Janne PA, Reblando J, Shrager S, Jaklitsch MT, Mentzer S, Lukanich JM, Sugarbaker DJ, Baldini EH, Berman S, Skarin A, Bueno R (2005) High risk of brain metastases in surgically staged IIIA non-small-cell lung cancer patients treated with surgery, chemotherapy, and radiation. J Clin Oncol 23(7):1530-1537. https://doi.org/10.1200/JCO.2005.04.123

5. Peters S, Camidge DR, Shaw AT, Gadgeel S, Ahn JS, Kim DW, Ou SI, Perol M, Dziadziuszko R, Rosell R, Zeaiter A, Mitry E, Golding S, Balas B, Noe J, Morcos PN, Mok T, Investigators AT (2017) Alectinib versus crizotinib in untreated ALK-positive nonsmall-cell lung cancer. N Engl J Med 377(9):829-838. https://doi. org/10.1056/NEJMoa1704795

6. Puhalla S, Elmquist W, Freyer D, Kleinberg L, Adkins C, Lockman P, McGregor J, Muldoon L, Nesbit G, Peereboom D, Smith Q, Walker S, Neuwelt E (2015) Unsanctifying the sanctuary: challenges and opportunities with brain metastases. Neuro-Oncology 17(5):639-651. https://doi.org/10.1093/neuonc/nov023

7. Mansfield AS, Aubry MC, Moser JC, Harrington SM, Dronca RS, Park SS, Dong H (2016) Temporal and spatial discordance of programmed cell death-ligand 1 expression and lymphocyte tumor infiltration between paired primary lesions and brain metastases in lung cancer. Ann Oncol 27(10):1953-1958. https://doi. org/10.1093/annonc/mdw289

8. Margolin K, Ernstoff MS, Hamid O, Lawrence D, McDermott D, Puzanov I, Wolchok JD, Clark JI, Sznol M, Logan TF, Richards J, Michener T, Balogh A, Heller KN, Hodi FS (2012) Ipilimumab in patients with melanoma and brain metastases: an open-label, phase 2 trial. Lancet Oncol 13(5):459-465. https://doi. org/10.1016/S1470-2045(12)70090-6

9. Goldberg SB, Gettinger SN, Mahajan A, Chiang AC, Herbst RS, Sznol M, Tsiouris AJ, Cohen J, Vortmeyer A, Jilaveanu L, Yu J, Hegde U, Speaker S, Madura M, Ralabate A, Rivera A, Rowen E, 
Gerrish H, Yao X, Chiang V, Kluger HM (2016) Pembrolizumab for patients with melanoma or non-small-cell lung cancer and untreated brain metastases: early analysis of a non-randomised, open-label, phase 2 trial. Lancet Oncol 17(7):976-983. https://doi. org/10.1016/S1470-2045(16)30053-5

10. Sloot S, Chen YA, Zhao X, Weber JL, Benedict JJ, Mule JJ, Smalley KS, Weber JS, Zager JS, Forsyth PA, Sondak VK, Gibney GT (2018) Improved survival of patients with melanoma brain metastases in the era of targeted BRAF and immune checkpoint therapies. Cancer 124(2):297-305. https://doi.org/10.1002/cncr.30946

11. Besse B, Le Moulec S, Mazieres J, Senellart H, Barlesi F, Chouaid C, Dansin E, Berard H, Falchero L, Gervais R, Robinet G, Ruppert AM, Schott R, Lena H, Clement-Duchene C, Quantin X, Souquet PJ, Tredaniel J, Moro-Sibilot D, Perol M, Madroszyk AC, Soria JC (2015) Bevacizumab in patients with nonsquamous non-small cell lung cancer and asymptomatic, untreated brain metastases (BRAIN): a nonrandomized, phase II study. Clin Cancer Res 21(8):1896-1903. https://doi.org/10.1158/1078-0432. Ccr-14-2082

12. Lu YS, Chen TW, Lin CH, Yeh DC, Tseng LM, Wu PF, Rau KM, Chen BB, Chao TC, Huang SM, Huang CS, Shih TT, Cheng AL (2015) Bevacizumab preconditioning followed by Etoposide and Cisplatin is highly effective in treating brain metastases of breast cancer progressing from whole-brain radiotherapy. Clin Cancer Res 21(8):1851-1858. https://doi.org/10.1158/1078-0432. Ccr-14-2075

13. Sandler A, Gray R, Perry MC, Brahmer J, Schiller JH, Dowlati A, Lilenbaum R, Johnson DH (2006) Paclitaxel-carboplatin alone or with bevacizumab for non-small-cell lung cancer. N Engl J Med 355(24):2542-2550. https://doi.org/10.1056/NEJMoa061884

14. Reck M, von Pawel J, Zatloukal P, Ramlau R, Gorbounova V, Hirsh V, Leighl N, Mezger J, Archer V, Moore N, Manegold C (2009) Phase III trial of cisplatin plus gemcitabine with either placebo or bevacizumab as first-line therapy for nonsquamous non-small-cell lung cancer: AVAil. J Clin Oncol 27(8):12271234. https://doi.org/10.1200/JCO.2007.14.5466

15. Ilhan-Mutlu A, Osswald M, Liao Y, Gommel M, Reck M, Miles D, Mariani P, Gianni L, Lutiger B, Nendel V, Srock S, PerezMoreno P, Thorsen F, von Baumgarten L, Preusser M, Wick W, Winkler F (2016) Bevacizumab prevents brain metastases formation in lung adenocarcinoma. Mol Cancer Ther 15(4):702710. https://doi.org/10.1158/1535-7163.MCT-15-0582

16. De Braganca KC, Janjigian YY, Azzoli CG, Kris MG, Pietanza MC, Nolan CP, Omuro AM, Holodny AI, Lassman AB (2010) Efficacy and safety of bevacizumab in active brain metastases from non-small cell lung cancer. J Neurooncol 100(3):443-447. https://doi.org/10.1007/s11060-010-0200-2

17. Berghoff AS, Sax C, Klein M, Furtner J, Dieckmann K, Gatterbauer B, Widhalm G, Rudas M, Zielinski CC, Bartsch R, Preusser M (2014) Alleviation of brain edema and restoration of functional independence by bevacizumab in brain-metastatic breast cancer: a case report. Breast Care (Basel) 9(2):134-136. https://doi.org/10.1159/000360930

18. Winkler F (2019) Anti-angiogenics in brain metastases: perspectives and experiences. In: Marmé D (ed) Tumor angiogenesis: a key target for cancer therapy. Springer, New York

19. Slotman B, Faivre-Finn C, Kramer G, Rankin E, Snee M, Hatton M, Postmus P, Collette L, Musat E, Senan S (2007) Prophylactic cranial irradiation in extensive small-cell lung cancer. N Engl J Med 357(7):664-672. https://doi.org/10.1056/NEJMoa071780

20. Gore EM, Bae K, Wong SJ, Sun A, Bonner JA, Schild SE, Gaspar LE, Bogart JA, Werner-Wasik M, Choy H (2011) Phase III comparison of prophylactic cranial irradiation versus observation in patients with locally advanced non-small-cell lung cancer: primary analysis of radiation therapy oncology group study RTOG 0214. J Clin Oncol 29(3):272-278. https://doi. org/10.1200/jco.2010.29.1609

21. Lynch M (2019) Preservation of cognitive function following whole brain radiotherapy in patients with brain metastases: complications, treatments, and the emerging role of memantine. J Oncol Pharm Pract 25(3):657-662. https://doi. org/10.1177/1078155218798176

22. Wolfson AH, Bae K, Komaki R, Meyers C, Movsas B, Le Pechoux C, Werner-Wasik M, Videtic GM, Garces YI, Choy H (2011) Primary analysis of a phase II randomized trial radiation therapy oncology group (RTOG) 0212: impact of different total doses and schedules of prophylactic cranial irradiation on chronic neurotoxicity and quality of life for patients with limited-disease small-cell lung cancer. Int J Radiat Oncol Biol Phys 81(1):77-84. https://doi.org/10.1016/j.ijrobp.2010.05.013

23. Kienast $\mathrm{Y}$, von Baumgarten L, Fuhrmann M, Klinkert WE, Goldbrunner R, Herms J, Winkler F (2010) Real-time imaging reveals the single steps of brain metastasis formation. Nat Med 16(1):116-122. https://doi.org/10.1038/nm.2072

24. Feng PH, Chen KY, Huang YC, Luo CS, Wu SM, Chen TT, Lee CN, Yeh CT, Chuang HC, Han CL, Lin CF, Lee WH, Kuo CH, Lee KY (2018) Bevacizumab reduces S100A9-positive MDSCs linked to intracranial control in patients with EGFR-mutant lung adenocarcinoma. J Thorac Oncol 13(7):958-967. https://doi. org/10.1016/j.jtho.2018.03.032

25. Hanna NH, Kaiser R, Sullivan RN, Aren OR, Ahn MJ, Tiangco B, Voccia I, Pawel JV, Kovcin V, Agulnik J, Gaschler-Markefski B, Barrueco J, Sikken P, Schloss C, Kim JH (2016) Nintedanib plus pemetrexed versus placebo plus pemetrexed in patients with relapsed or refractory, advanced non-small cell lung cancer (LUME-Lung 2): a randomized, double-blind, phase III trial. Lung Cancer 102:65-73. https://doi.org/10.1016/j.lungc an.2016.10.011

26. Valiente M, Van Swearingen AED, Anders CK, Bairoch A, Boire A, Bos PD, Cittelly DM, Erez N, Ferraro GB, Fukumura D, Gril B, Herlyn M, Holmen SL, Jain RK, Joyce JA, Lorger M, Massague J, Neman J, Sibson NR, Steeg PS, Thorsen F, Young LS, Varešlija D, Vultur A, Weis-Garcia F, Winkler F (2020) Brain metastasis cell lines panel: a public resource of organotropic cell lines. Cancer Res. https://doi.org/10.1158/00085472.CAN-20-0291

27. Hilberg F, Roth GJ, Krssak M, Kautschitsch S, Sommergruber W, Tontsch-Grunt U, Garin-Chesa P, Bader G, Zoephel A, Quant J, Heckel A, Rettig WJ (2008) BIBF 1120: triple angiokinase inhibitor with sustained receptor blockade and good antitumor efficacy. Cancer Res 68(12):4774-4782. https://doi.org/10.1158/00085472.Can-07-6307

28. Schindelin J, Arganda-Carreras I, Frise E, Kaynig V, Longair M, Pietzsch T, Preibisch S, Rueden C, Saalfeld S, Schmid B, Tinevez JY, White DJ, Hartenstein V, Eliceiri K, Tomancak P, Cardona A (2012) Fiji: an open-source platform for biological-image analysis. Nat Methods 9(7):676-682. https://doi.org/10.1038/nmeth.2019

29. Winkler F, Kozin SV, Tong RT, Chae SS, Booth MF, Garkavtsev I, Xu L, Hicklin DJ, Fukumura D, di Tomaso E, Munn LL, Jain RK (2004) Kinetics of vascular normalization by VEGFR2 blockade governs brain tumor response to radiation: role of oxygenation, angiopoietin-1, and matrix metalloproteinases. Cancer Cell 6(6):553-563. https://doi.org/10.1016/j.ccr.2004.10.011

30. Palmieri D, Bronder JL, Herring JM, Yoneda T, Weil RJ, Stark AM, Kurek R, Vega-Valle E, Feigenbaum L, Halverson D, Vortmeyer AO, Steinberg SM, Aldape K, Steeg PS (2007) Her-2 overexpression increases the metastatic outgrowth of breast cancer cells in the brain. Cancer Res 67(9):4190-4198. https://doi. org/10.1158/0008-5472.Can-06-3316

31. Yuan A, Yang PC, Yu CJ, Lee YC, Yao YT, Chen CL, Lee LN, Kuo SH, Luh KT (1995) Tumor angiogenesis correlates with 
histologic type and metastasis in non-small-cell lung cancer. Am J Respir Crit Care Med 152(6 Pt 1):2157-2162. https://doi. org/10.1164/ajrccm.152.6.8520790

32. Berghoff AS, Ilhan-Mutlu A, Wohrer A, Hackl M, Widhalm G, Hainfellner JA, Dieckmann K, Melchardt T, Dome B, Heinzl H, Birner P, Preusser M (2014) Prognostic significance of Ki67 proliferation index, HIF1 alpha index and microvascular density in patients with non-small cell lung cancer brain metastases. Strahlenther Onkol 190(7):676-685. https://doi.org/10.1007/s0006 6-014-0639-8

33. Berghoff AS, Ilhan-Mutlu A, Dinhof C, Magerle M, Hackl M, Widhalm G, Hainfellner JA, Dieckmann K, Pichler J, Hutterer M, Melchardt T, Bartsch R, Zielinski CC, Birner P, Preusser M (2015) Differential role of angiogenesis and tumour cell proliferation in brain metastases according to primary tumour type: analysis of 639 cases. Neuropathol Appl Neurobiol 41(2):e41-55. https://doi.org/10.1111/nan.12185

34. Berghoff AS, Breckwoldt MO, Riedemann L, Karimian-Jazi K, Loew S, Schlieter F, Furtner J, Cinci M, Thomas M, Strowitzki MJ, Marmé F, Michel LL, Schmidt T, Jäger D, Bendszus M, Preusser M, Wick W, Winkler F (2020) Bevacizumab-based treatment as a salvage therapy in patients with recurrent symptomatic brain metastases. Neuro-Oncol Adv. https://doi.org/10.1093/noajn $1 / \mathrm{vdaa} 038$

35. Berghoff AS, Schur S, Fureder LM, Gatterbauer B, Dieckmann K, Widhalm G, Hainfellner J, Zielinski CC, Birner P, Bartsch R, Preusser M (2016) Descriptive statistical analysis of a real life cohort of 2419 patients with brain metastases of solid cancers. ESMO Open 1(2):e000024. https://doi.org/10.1136/esmoopen2015-000024

36. Xu Q, Zhang Z, Chen Z, Zhang B, Zhao C, Zhang Y, Zhao C, Deng X, Zhou Y, Wu Y, Gu J (2019) Nonspecific immunoglobulin $\mathrm{G}$ is effective in preventing and treating cancer in mice. Cancer Manage Res 11:2073-2085. https://doi.org/10.2147/cmar.S1881 72

37. Krause I, Shoenfeld Y (2005) Intravenous immunoglobulin treatment for fibrosis, atherosclerosis, and malignant conditions. Methods Mol Med 109:403-408. https://doi.org/10.1385/1-59259 $-862-5: 403$

38. Sobieszczanska M, Tubek S, Poplicha D, Grabelus A, Pawelczak J (2014) Henoch-Schonlein purpura (HSP) and high-dose immunoglobulin treatment in patient with familiar prostatic adenocarcinoma. Human Vaccines Immunother 10(2):358-359. https://doi. org/10.4161/hv.27012

39. Subhadra C, Dudek AZ, Rath PP, Lee MS (2008) Improvement in visual fields in a patient with melanoma-associated retinopathy treated with intravenous immunoglobulin. J Neuro-Ophthalmol 28(1):23-26. https://doi.org/10.1097/WNO.0b013e31816754c4

40. Murie-Fernandez M, Gurpide A, de la Cruz S, de Castro P (2006) Total remission of thymus carcinoma after treatment with intravenous immunoglobulin. Clin Transl Oncol 8(9):697-699. https ://doi.org/10.1007/s12094-006-0043-7
41. Corp. SA (2002) Normal serum, ascites, and cell supernatant typical immunoglobulin concentration ranges normal sera. https ://www.sigmaaldrich.com/content/dam/sigma-aldrich/docs/Sigma /General_Information/normalimmunoranges.pdf. Accessed 04 Jan 2019

42. Damianovich M, Blank M, Raiter A, Hardy B, Shoenfeld Y (2009) Anti-vascular endothelial growth factor (VEGF) specific activity of intravenous immunoglobulin (IVIg). Int Immunol 21(9):10571063. https://doi.org/10.1093/intimm/dxp070

43. Shoenfeld Y, Fishman P (1999) Gamma-globulin inhibits tumor spread in mice. Int Immunol 11(8):1247-1252

44. Dominguez-Soto A, de Casas-Engel M, Bragado R, MedinaEcheverz J, Aragoneses-Fenoll L, Martin-Gayo E, van Rooijen N, Berraondo P, Toribio ML, Moro MA, Cuartero I, Castrillo A, Sancho D, Sanchez-Torres C, Bruhns P, Sanchez-Ramon S, Corbi AL (2014) Intravenous immunoglobulin promotes antitumor responses by modulating macrophage polarization. J Immunol 193(10):5181-5189. https://doi.org/10.4049/jimmunol.13033 75

45. Hofmann I, Baum A, Hilberg F, Garin Chesa P, Depla E, Boucneau J, Kraut N, Künkele K-P (2015) Dual targeting of angiogenesis pathways: combined blockade of VEGF and Ang2 signaling. 8th Euro Global Summit on Cancer Therapy, Valencia, Spain

46. Bohn KA, Adkins CE, Nounou MI, Lockman PR (2017) Inhibition of VEGF and angiopoietin-2 to reduce brain metastases of breast cancer burden. Front Pharmacol 8:193. https://doi.org/10.3389/ fphar.2017.00193

47. Steeg PS, Theodorescu D (2008) Metastasis: a therapeutic target for cancer. Nat Clin Pract Oncol 5(4):206-219. https://doi. org/10.1038/ncponc1066

48. Anderson RL, Balasas T, Callaghan J, Coombes RC, Evans J, Hall JA, Kinrade S, Jones D, Jones PS, Jones R, Marshall JF, Panico MB, Shaw JA, Steeg PS, Sullivan M, Tong W, Westwell AD, Ritchie JWA (2019) A framework for the development of effective anti-metastatic agents. Nat Rev Clin Oncol 16(3):185-204. https ://doi.org/10.1038/s41571-018-0134-8

49. Steeg PS (2016) Targeting metastasis. Nat Rev Cancer 16:201. https://doi.org/10.1038/nrc.2016.25

50. Reck M, Kaiser R, Mellemgaard A, Douillard JY, Orlov S, Krzakowski M, von Pawel J, Gottfried M, Bondarenko I, Liao M, Gann CN, Barrueco J, Gaschler-Markefski B, Novello S (2014) Docetaxel plus nintedanib versus docetaxel plus placebo in patients with previously treated non-small-cell lung cancer (LUME-Lung 1): a phase 3, double-blind, randomised controlled trial. Lancet Oncol 15(2):143-155. https://doi.org/10.1016/s1470 $-2045(13) 70586-2$

Publisher's Note Springer Nature remains neutral with regard to jurisdictional claims in published maps and institutional affiliations. 